\title{
God, Mind, Evolution, and Quantum Reality Based on Process Metaphysics
}

\section{Mark Germine*}

You cannot shelter theology from science, or science from theology; Nor can you shelter either from metaphysics, or metaphysics from either of them.

There is no short cut to truth.

Alfred North Whitehead (1927, p. 79)

Abstract

The genesis of actuality from potentiality, with the apparent role of the observer, is an important and unsolved problem which essentially defines science's view of reality in a variety of contexts. Observation then becomes lawful and not emergent. Panentheism is needed to provide a mechanism for order outside of blind efficient causality, in a Universal final causality. Classical physics is over a hundred years out of date, yet scientific models remain mechanistic and deterministic. Deism, a remnant of classical cosmology, is examined and rejected by scientists and philosophers, and certain pre-scientific notions of religion are scorned, putting the matter to rest. Quantum physics, in its basic form, is necessary if there is to be any philosophical or scientific notion of free will and self-determination, as potentiality. Quantum metaphysics is also needed because classical physics is fundamentally

*germinemark@gmail.com 
limited to localized external relations, lacking the internality and non-locality of relatedness. God, or the equivalent, is necessary to complete the equation. Physicists now tell us that reality is fundamentally mental and is created by observation. Observation is here taken to mean experience, with experience going all the way down to the lowest order of a Universal mentality.

\section{Introduction}

Of late we have seen a number of books that allege that science has disproved the existence of God. Often such books refer to the Darwinian theory of evolution by natural selection as the final word on the phenomenon of man. As a child psychiatrist, I once asked a child if he believed in God. His answer was: "No, I believe in evolution." The notion that the existence of God and the general theory of evolution are mutually exclusive seems to have penetrated the very fabric of society. Darwin has, in a sense, become the prophet of a new religion, "survival of the fittest." We will argue here that evolution does not exclude the agency of God, but, in fact, requires God.

The theory of mind is a burgeoning field, and, following a form of philosophy called eliminative materialism, it pretends to explain mind by "eliminating" or discounting subjective experience. God has been banished from science, with the justification that science must be naturalistic, while God is said to be supernatural. Scientists are increasingly identifying themselves as atheist, as are students of science, and the lay public that has been influenced by such science. Those who have not embraced the "scientific" dogma of atheism, and who believe in God, generally give no place for God in science, and often hold two separate and mutually exclusive belief systems, a spiritual one and a scientific one.

Process theory, particularly the metaphysics of Alfred North Whitehead, gives us a natural, relational theology in which God does not simply sit idly by and watch the world go round, but is an active participant, indeed a necessary predicate, to any scientific view of reality. The explanatory power of the major scientific theories of physics, mind, and evolution have reached a limit, and 
much of atheistic science has become a collection of leaps of faith in the dogma of neo-classical, materialist fundamentalism.

The focus of this paper is on a process view of mind, brain, and mentality in general. We will argue here that function of the mind involves final causation or teleology. As stated by Whitehead (1978, p. 277):

"The mental operations have a double office. They
achieve, in the immediate subject, the subjective aim
of that subject as to the satisfaction to be obtained
from its initial data. In this way the decision derived
from the actual world, which is the efficient cause, is
completed by the decision embodied in the
subjective aim, which is the final cause...Thus the
mental pole is the link whereby creativity is
endowed with the double character of final
causation, and efficient causation."

Whitehead further tells us that mentality is non-spatial (1978, p. 108), or non-local, if one uses the quantum term, and, by implication, non-temporal. The process of mind can be viewed as a cycle involving the conceptual or mental pole and the physical pole. This would be consistent with Whitehead's assertions (1978, p. 348): "For God the conceptual is prior to the physical, for the World the physical poles are prior to the conceptual poles...God is the infinite ground of all mentality."

Mind/matter dualism, in the Cartesian sense, posits a substantial mind or "mind stuff." There is not a shred of evidence that such stuff exists, or that the mind is substantial. Whitehead's panexperentialism makes experience primary, as it should be in science. All we know is based on experience. The actual occasion is, fundamentally, a quantum of experience. The existence of matter is implied by experience. We also think of matter as "stuff," but have no firm evidence beyond our senses that this is the case. Entities that are thought of as substantial are really relational, and all of empirically-derived science is based on relations. 


\section{The Brain and the Mind}

The agenda here is to examine the process of mentality in a process sense, with a focus on Whitehead and PR. Whitehead's concept of qaunta of experience flows directly from the emergence of quantum theory, but efforts to bridge this connection between experience and quantum physics in process metaphysics is far from completion. The fulfillment of this effort must include the functions of the human brain, as well as the role of quantum processes. Classical physics, which is incurably mechanistic and deterministic, cannot support the notions of free will and selfdetermination, nor, for that matter, can it truly support process metaphysics. In a sense, Whitehead seemed to recognise the need for a broader approach to process (1978, pp. 238-9): “Modern physicists see energy transferred in definite quanta. This quantum theory also has analogues in recent neurology." There has been enormous progress in the field of neurology and neuroscience, as well as physics, since Whitehead's time, and it is important for us to bring such progress up to date in terms that are applicable to process metaphysics.

Many types of quantum indeterminacy exist in the brain (e.g. Stapp), and each type has been theorized as the basis of consciousness, which bears upon the nature of experience. The problem with all these kinds of uncertainty is that they are purely physical. The recurrent action of subjective or conceptual process is needed in order to achieve a reiterative becoming of levels of progressively higher orders of mental function, and this process includes the agency of the person or Self, which is derivative of the One Mind or Mind of God. As God is the source of all mentality, this process is not purely one of efficient causality in the physical processes that are generated by quantum uncertainty, with subjectivity emerging without reference to a subject. The subjective aim brings the subject into the process of mentality. The subjective aim is teleological, in that recursive processes of conceptual prehension have physical prehensions as their data.

Here we accept the underlying summation of all quantum indeterminacy, which collectively would cause bifurcation of mental states in an indeterminate fashion. On this basis, the level of quantum uncertainty is such that the unfolding of dynamical, 
chaotic processes develops into a large number of virtual or potential mental states. The reliance on the individual, physical brain in the emergence of mentality is vastly underdetermined. Final causation is necessary to supply the subjective aim, with God as the source, functioning on the basis of a universal knowledge. Without the action of a universal process, there is no solution to the binding problem. Binding allows the entire brain to be internally connected in such way as to constitute a single, living person. The action of neural processing, transmission, and integration is otherwise fragmented, and far too slow to provide a cohesive entity.

The universal knowledge that is needed for a realistic view of the functioning of the individual mind has many references in process theory, which bring the role of the universe into the becoming. This is summed up, in part, as follows (Whitehead, 1978, p. 165): "The concrescence is an individualization of the whole universe." The Universe spans billions of light years, such that in the participation of every item or event in the Universe into each cycle of becoming does not seem possible in a physically relativistic Universe. Rather than bringing in some physical process into the realm of subjectivity and Mind, we posit a Universal Mentality. The agency of One Mind includes the entire universe, as it is extended in both space and time the doctrine of panentheism. The "individual person" is part of a society of occasions, which enters into a constitution that includes all occasions in the entire universe. The prehensions thereof enter into the consequent nature of God, in keeping with the One Mind Model.

Science cannot endorse the impossible, or enter into fanciful theories of a holography based on what is purely physical. The mind is of such nature that it could not possibly work on the sole basis of localization of physical processes. The One Mind Model, in addition to the place it may take in metaphysics, is a legitimate theory of quantum reality in physics. This model has received some validation in principle based on experimental work using a form of electroencephalography called Event Related Potentials (Germine, 2011).We will later address other models of quantum reality.

There seems to be a relation between mind and energy, which Whitehead expressed as follows. Whitehead (1968, p. 168): “...the 
operation of mentality is primarily to be conceived as a diversion of the flow of energy...the energetic activity considered in physics is the emotional intensity entertained in life." The living brain is always far from equilibrium, and as such, it is a dynamical system that is upheld by the action of expending free energy, which is part of its metabolism. This energy flows on the basis of a shifting of blood supply, providing energy to uphold the shifting activation of various parts of the brain. Emotions are sub-served primarily by the limbic system. By-and-large, the limbic system is shared with other mammals, and as such, other mammals are subjective in the physical sense, maintaining conceptual feelings only in a rudimentary way. The hypertrophied cerebral cortices of the brains of humans modulating the intensity of emotions, involving both facilitation and inhibition of emotional tone. The expenditure of energy, then, is an ingredient to emotional energy, but can involve facilitation as well as inhibition of emotional intensity.

In the structured inhibition of emotion, there is an involution of the internal relations between people and with the world, and the brain/mind comes to be viewed as a prison in the cranium, with no actual contact with others beyond what we perceive in the brain, capable of functioning with no need for God. This is the leap of faith that nearly all of modern science has taken, as well as the existential crisis that looms upon our world and humankind. The person, being in the likeness of God, rarely enters the scientific equation.

Most theories of mind and consciousness assume that they are emergent phenomena, yet there is no real evidence in theory or in experimental fact to guide us as to how they emerge. The processes in the brain are qualitatively different from what we experience. The processes in the brain involve chemical interactions and electrical activity, which, on a materialistic basis, do not translate into experience. Specific functions are associated with certain regions of the brain, and neuroscience is busy identifying these regions, but the unity and quality of experience have escaped scientific explanation. 
Conscious experience is taken up here as the leading edge of a progressive process that has its source in unconscious experience, which goes all the way down to the level of the most rudimentary experience. However, the theory of the unconscious has been abandoned by the larger scientific community. In the science of psychology, behaviorism is embraced progressively, making the person into a kind of non-entity, a "black box," that is ruled by conditioning.

The brain has been considered by many to have a quantum wave function that is "collapsed," in the sense that the wave function of the brain is in a virtual superposition of states. The idea that the brain has a distinct quantum "wave function" of superimposed states that "collapses" is the cause and/or result of consciousness has lately fallen from favor as a result of the decoherence model. Decoherence would then transform quantum events in the brain into quasi-classical states of the brain by interaction with the physical environment. The collapse model holds that this collapse evokes consciousness through the agency of von Neumann's (1954) "abstract ego," placing individual human conscious experience in a position of being the arbiter of physical reality. The ego, as arbiter of reality, would then be ego-consciousness, which evokes consciousness by observation in discrete intervals.

There are several problems with this approach. These problems, their possible solution in a theory of universal mind, and the dismissal of such a solution were described by Bohm and Hiley (1993, pp. 23-24):

"We can see several difficulties in the attempt to bring in the direct action of the mind to give an ontological interpretation of the current physical laws of the quantum theory. Thus, in the laboratory, it is hard to believe that the human mind is actually significantly affecting the results of the functioning of the instruments. Moreover quantum theory is currently applied to cosmology, and it is difficult to believe that the evolution of the universe before the appearance of human beings depended fundamentally on the human mind (e.g. to make its wave function 'collapse' in an appropriate way). Of 
course one could avoid this difficulty by assuming a universal mind. But if we know little about the human mind, we know a great deal less about the universal mind."(Italics added)

Universal Mind is a solution to a great dilemma in science's quest for a model of quantum reality. In an observer-created reality, what is potential becomes actual. If there were more than One Mind, each individual would be governing its own universe, private and separate from others. The models based on divergent universes of quantum reality are called the "many worlds," and the "many minds" models, which are still the most widely accepted models of quantum reality in physics. However, our experience tells us that all minds are part of the same world. The One Mind is one of the many names of God. This concept does not negate the individual mind, but it does qualify it. There is no mind outside of the agency of God, whether God is viewed as the society of Hartshorne or the actual entity of Whitehead. This One Mind is everywhere present through all of time and space. It makes the multiplicity of the potential one actuality, whether it be in the genesis of the Universe out of all possible universes, the selection of a particular universe out of parallel universes, or in the reduction of quantum uncertainty in observation. The One Mind Model of quantum reality would thus entertain a variety of current theories with equal ease.

Indeterminacy becomes determinate in the concrescence (Whitehead, 1978, p. 23). It is God who supplies the initial subjective aim for all actualities, the appetition that moves towards satisfaction of the subjective aim in an epochal or discontinuous manner. Once this is realized through the temporal process of transition, the actuality in completed and passes into objective immortality. It is thus that God is "the principle of concretion," which produces an actuality from the field of potentiality. Such a process demands a quantum explanation, as "potentia" do not exist in classical physics, but only in quantum physics. Beyond this, it demands a dynamical system when applied to the human brain, and to those creatures who partake of the "creativity," as we will we later discuss. 
Current quantum models of brain process all suffer from the same inadequacy, and that is that quantum uncertainty is, for all intents and purposes, limited to the microscopic realm. The brain, in and of itself, is not a quantum object. Thus, the very existence of freewill has been denied, and consciousness is widely considered to be epiphenomenal, a by-product of the brain that his no function or volitional component. Assuming evolution by natural selection, one would have to question why consciousness would develop if it serves no purpose.

The living brain is a dynamical system, meaning that it is far from equilibrium at all times, and dissipates energy. Such systems are called chaotic, not in the sense that they are disordered, but in mathematical parlance. Chaotic systems, or systems at the "edge of chaos and order," such as the brain, exhibit the property of selforganisation, or self-organising criticality. The criticality lies in the exquisite sensitivity of the state of the system to small changes in physical conditions.

As a dynamical system, the brain, as per current mainstream neuroscience, would produce unpredictable states, much as the weather cannot be predicted, with all of our current technology. Given current conditions, the state of the system would become increasingly unpredictable with the passage of time. If one introduces quantum theory with all its uncertainties, the brain state would become even more unpredictable. This unpredictability would totally compromise the rational function of mental process, memory, and our sense of an abiding identity.

The only possible way out of this conundrum, without resorting to a mind/brain dualism, is to posit a non-temporal process which flows from the mental to the physical, where the final cause or teleology of the state of mind becomes the subjective aim, the reason for its own becoming according to the ontological principle (PR). In this case, the combination of self-organising criticality and quantum variability, as applied globally over a period of time, with the sum of quantum events being amplified by chaotic dynamics, is distilled in the action of the subjective aim such as to bring about the final cause. Efficient causality in time would then be under the influence of final causality, operating outside of time, and essentially guiding mental process. The only possible agent of such 
an influence would be God, as described by Whitehead previously, or what we have called Self, God within us, which we will examine shortly. Causality is not violated, since the future does not affect the past, which is already settled.

Metaphysics in a relativistic Universe, requires the future to be real, albeit as potential. The potentials are reduced over time in that these potentials are contingent on the basis of relation between time and causality. Thus, for example, it might be possible that I would be at a remote location five minutes from now may not be possible, it may have been possible yesterday, but unlikely. If I had planned to be in this remote locality two weeks ago, it would become a potential that is likely to actualize as planned.

A plurality of possible universes exists in the future, and God knows them all, but we, as creatures, do not. The knowledge of future potentials makes the subjective aim and final causality work, and requires God as the "ground" of mentality. A panentheistic approach allows this to be the case. In God and the World we have a mutual transcendence. The process works within the realm of possibility or potentiality. If we are to bring science and religion together, then on the side of science we must abandon the concept of the supernatural. If something happens, from the scientific point of view, it is possible. This does not make God impossible, or imply that God's role in the creation and a progressive mutual transcendence does not occur between God and the World.

\section{Mind and Matter}

It is widely assumed that matter, through a complex system such as the brain, gives rise to mind. This assumption is problematic in that it makes mind, and by implication spirit, emergent from matter and not a fundamental actuality. This is the theory of emergence, which is currently the mainstream theory of mind in science. Whitehead attributes experience and subjectivity to the most fundamental actual entities. Actual entities can have intellectual operations and be conscious, as Whitehead states (Whitehead, 1978, p. 326): 


\begin{abstract}
"The complex of such intellectual operations is sometimes termed the 'mind' of the actual occasion; and the actual occasion is also termed 'conscious.' But the term conveys the suggestion of independent substance. This is not meant here: a better term is the 'consciousness' belonging to the actual occasion."
\end{abstract}

The ego is also considered by Whitehead to be an actual entity. Whitehead addresses this in his refutation of Cartesian substantial dualism (1978, p. 75): "For each time he pronounces 'I am, I exist,' the actual occasion, which is the ego, is different; and the 'he' which is common to the two egos is an eternal object or, alternately, the nexus of successive occasions." Adopting the former alternative, that the 'he' that is shared by the two egos is an eternal object, we note that (Whitehead, 1978, p. 23) "eternal objects are the same for all actual entities," implying that this 'he' can have ingression in the becoming of occasions that are totally removed from the single individual. Furthermore, Whitehead defines the eternal object as follows: (Whitehead, 1978, p. 44) "Any entity whose conceptual recognition does not involve a necessary reference to any definite actual entities in the temporal world is called an 'eternal object."'

The ego, as an eternal object, would then have to be considered to be a kind of form of mentality that is not particular to any individual. This construction of the ego is perhaps a matter of semantics, but the ego as generally conceived in psychology, has reference to the temporal world. From a psychological perspective, and deriving from metapsychology, the Self is the primordial and unqualified "eternal object." In terms of the metalogic of religions, this is quite explicit in the Upanishads. The confusion between Islam and Christianity, which is troubling our world, is created when the Self becomes God, as in Christianity. There are many names for God, and many manifestations of God, when considered as One Mind.

The problem of the mind/matter duality arises as science grapples with the idea that mind arises out of matter. The importance of mind is trivialized as the physical functions of the brain are elaborated without reference to mind. There does not seem to be any empirical data explaining how consciousness arises in the 
brain, although, as far as we know empirically, the brain is the only system which manifests consciousness. Consciousness is seen as the end product of a process in the brain, through efficient causation, serving no known function. Conscious process must involve final causation in order for consciousness in the mind/brain to be intelligible.

In his discussion of the defining characteristics of living things, Whitehead states (1978, p. 104): “We require explanation by 'final cause.' Thus, the single occasion is alive when the subjective aim which determines its process of concrescence has introduced a novelty of definiteness not to be found in the inherited data of the primary phase." In Whitehead's cosmology, God supplies the subjective aim, and (1978, p. 67) "God is the organ of novelty, aiming at intensification." The novelty is in the becoming of experience through the final causation of the subjective aim, supplied by God. In relation to the World, God seeks a mutual transcendence, and the intensification of novelty in the World is the process of increasing "intensities" of experience.

It may be argued that this increasing intensity of experience over time holds the cause of our own existence, as well as that of our Universe and all living creatures. What is real can be described by the Zen Buddhist koan as: "the sound of one hand clapping." The one hand is the quantum field of possibilities. The observer of the field is the other hand. Neither hand alone makes a sound when moved as if to clap. Both hands make a sound, the field and the observer, or, to paraphrase the Bhagavad Gita, the knower of the field. Evolution then is impelled by the intensification of experience, while natural selection is just a permissive phenomenon.

In the matter of time within the quantum field as potential in the realm of the becoming and concrescence of the actual entity, we entertain this view of time in terms of Whitehead's concept of the extensive continuum, as a field of potentiality underlying all of space and all of time - past, present, and future. To quote Whitehead (1978, p. 72): 


\begin{abstract}
"The extensive continuum is that general relational element in experience whereby the actual entities experienced, and that unit experience itself, are united in the solidarity of one common world. The actual entities atomize it, and thereby make real what was antecedently merely potential. The atomization of the extensive continuum is also its temporalization; that is to say, the becoming of actuality into what in itself is merely potential."
\end{abstract}

Experience is what is real. Experience is also the quantum process that makes the potential actual. Actual entities "deposit" time in increments that become with the becoming of seriality of time, which is atomized in the sense that these units or atoms of experience are derived from a continuum in a common world. This is the "temporalization" ${ }^{1}$. The extensive continuum would seem to be the precursor of time. In this sense, it could be a kind of "primitive" time, a relational time. The becoming of the actual entity, as we have discussed, "intensifies" over a period of time. The extensive continuum, existing as potential, then would be the medium for this intensification to occur, through the dual process of temporalisation and actualisation.

In Whiteheadean process, there seem to be two worlds, and this is consistent through much of Whitehead's work (1926, p. 103): “A mental occasion is an ultimate fact in the spiritual world, just as a physical occasion of blind perceptivity is an ultimate fact in the physical world. There is an essential reference from one world to the other." In order for mentality to exist in a "spiritual world," the "spiritual world" must underlie the substance of the brain, as implied by panentheism, while exerting causal influence including final causation over the evolving mental state.

Based on a quantum model, potentials exist in the field of actuality. These potentials are not temporal, and not subject to change over time, since time is an actuality. What becomes actual, becomes through the non-temporal concrescense or microscopic process. Time does not enter the picture until the concrescence achieves

${ }^{1}$ We are aware that Bergson took exception to such a process. 
satisfaction, and only at this "time" does the transition, or in macroscopic process, comes into the picture. The becoming of the actual entity is then completed, and it passes into objective immortality. The actual entity, having passed on to objective immortality, is pure potential, as eternal object, is the fundamental basis and lure of the consequent chain of becoming. The process is described as follows (1978, p. 69):

\begin{abstract}
"The conclusion is that in every act of becoming there is the becoming of something with temporal extension; but the act itself is not extensive, in the sense that it is divisible into earlier and later acts of becoming which correspond to the extensive divisibility of what has become."
\end{abstract}

\title{
Recursion and Neural Networks
}

Brain science has come a long way in recent years, and scientists have found that, by modeling connectivity after processes in the brain, important advances have been made in computation. The very notion of local connectivity, in the manner of computation, fails to take the agency of the person and the subjectivity to the mind, but has some relevance here.

The notion of recurrent neural networks, RNNs, leads us to explore the notion of recursion in process metaphysics. The term "recursion" is used in the technical parlance, but we will consider "recursion" to be equivalent to "reversion" in process terms, differing in nuance. First, we will to address the relationship between the "physical" and the "conceptual" processes involved in mentality as it is developed in the human brain.

There is a part of the cerebral cortex of the human brain called Wernicke's area. This area is located in the left or dominant temporal lobe for the majority of people. Its function is the comprehension of language. Language is rich in concepts, which are mental in nature. If the substance of this area of the brain loses all function due to stroke, injury, or some other process, the individual will become completely unable to comprehend language. Thus, this physical function of the brain precedes the conceptual function. A language must be learned, such that the physical prehension acquires the data of the conceptual prehension. 
Applied to process, this combination of a physical prehension can, for example, in learning, acquire the data of a conceptual prehension, making it a hybrid prehensionas follows (Cobb, 2008, pp. 42-43):

"Once Whitehead recognized that physical
prehensions could have conceptual prehensions as
their data, he had a new way of understanding cells
and of persons as well as much else. The novelty
introduced into one occasion by reversion can be
transmitted to the next. The novel feeling of the past
occasion becomes part of the physical prehension of
the new occasion and can be transmitted to future
occasions through pure physical prehensions. If
novel feelings could not be felt thereafter by hybrid
prehensions, they could have no continuing effect."

In other words, the physical prehension can engender a conceptual prehension, but, as such, the physical prehension becomes the source of a hybrid prehension, and the conceptual feeling continues in subsequent occasions as a hybrid feeling, "inheriting" its causal physical nature. The process of reversion brings the conceptual prehension to bear on an antecedent physical prehension. This seems to be the way that concepts arise in the mind from the physical stratum beneath it in the brain.

Recurrent neural networks (RNNs) are often described in computational terms, with reference to the brain, from which they were developed. Dynamical systems theory, both continuous and discontinuous, has become linked with RNNs in a variety of ways. An important feature in terms of process is the concatenation of back-propagated and feed-forward processes, thereby creating feedback loops that have an important function in the memory processes of RNNs. These short and long term processes allow the process of replication and contextualisation in the antecedent and subsequent features in the neural network "time step" of the current occasion of becoming.

The combination of recursion of former actualities and progression into subsequent actualizations includes both physical and mental processes. This is a process of "binding" of the mental state in it 
function of short and long term memory. It is such binding, taking place globally in the brain that allows the mind/brain states to be their process of becoming in sequential integration of a complex nature. This integration includes words in language, in notes in both melody and harmony in music, in emotional reversion and progression, and in a long list of other phenomena. This integration also includes all of these various fields of experience, such that there is also a process of integration through various modalities.

In this sense of progression and reversion, we may consider the nature of the concept of vibrational processes (1978, p. 279) to take an important role of an enduring society of occasions (1978, p. 34). In terms of the social order, the nexus of social and personal order takes on the feature of endurance, as "the nexus forms a single line of inheritance of its defining characteristic. Such a nexus is an 'enduring object.' It might have been terms a 'person,'..." (1978, pp. 34-35). The nature of the term "person" is qualified: "The nexus 'sustains a character' and this is one of the meanings of the Latin word 'persona'” (1978, p. 35)

Various models of RNNs involve recurrent feedback loops with back-propagation with feed-forward actions in time steps. Our description here involves one such model (Trischler \& D' eleutero, 2016), which synthesizes previous models. Briefly, regarding neural network theory and practice, recurrence involves loops in times steps of connected neurons, which exhibit qualities of dynamical systems, including vector fields and chaotic attractors. This produces a feedback loop going backward and forward within the single time step, which is then passed on to the next time step. There is progression and recurrence between network time steps, which are intricately related in the concatenation of the progressive unfolding of concepts and thoughts, as applied to the brain. The recurrence or recursion of experience involves the partial replication of the antecedent time step, with some difference evolving, and with some replication in the development of subsequent time steps. The process may involve multiple recurrences of the series of time steps, with a progressive development.

This sort of process is described in the context of process metaphysics (Cobb, 2008, p. 37). What is described an example of 64 
being irritated by what someone says. The tendency is to reproduce the irritation in the next moment. Although it is suggested that the irritation is a possibility, if one is irritated to the point of intense anger, most people will remain angry for some time, thus the need to remove oneself from a situation to "cool down." Cobb goes on to write that one may later experience the irritation, but the irritation may be compounded by embarrassment or shame. This would then constitute a reversion to the former irritation, but not exactly of the same type. The same kind of process can repeat itself for quite some time, evolving over time, and with the potential to go so far as to "explode" in magnitude, or, on the other end of the spectrum, to "shrink," and the process may be terminated. We then say that our emotional episode is "forgotten," not in the sense that one forgets the episode itself, but in the sense that the memory no longer elicits the emotion engendered during the episode. In the former sense we have a continuing. In both cases the process may be "saved" by long-term potentiation in the brain. The same kind of dynamics may be applied to Love, which is a very special case. Among all emotions, it is love that most "transcends" the ego, and involves the agency of Self, God within us. Love is the essence of harmony, and, in this sense, "God is Love." God is said to "Love the World.' In this sense God transcends the World. The mutual transcendence is that the World loves God. There is nothing more to say.

Harmony, not competition, is the predominant evolutionary force which God actualizes in nature (Whitehead, 1926, p. 156). The Self arises as a reality in the brain, fundamentally, as a place for God to realize that harmony. This Self, together with the evolving brain, has its highest purpose in unity with God. The unity of Self/God is the same as the Atman/Brahman unity in eastern religion.

We are in this Universe for a reason, to live in harmony. We are a species very early in our evolution, and our future is uncertain, but there is no way to stop this progressive harmonization, which is a final cause in the Mind of God, which we call the One Mind. The One Mind Model of quantum reality essentially holds that the One Mind manifests itself in nature as a single, actual Universe evolving over time over a particular trajectory. The Universe, as per Charles 
Hartshorne, is the body of God. Actual entities then would all belong to this Universal organism.

Albert Einstein (1950) held that our separateness from the Universe and others, which we ascribe to the ego, is a delusion, as in the following famous quotation:

"A human being is a part of the whole, called by us,
"Universe," a part limited in time and space. He
experiences himself, his thoughts and feelings as
something separated from the rest -- a kind of
optical delusion of his consciousness.
This delusion is a kind of prison for us, restricting us
to our personal desires and to affection for a few
persons nearest to us."

\section{Evolution}

The evolution of life on earth is a scientific fact, which would be futile to argue from any perspective. Charles Darwin developed the theory of evolution by natural selection. In doing so he made a major assumption, which we challenge here, and that is that there is no final causation in evolution.

Natural selection is like a ticket to board the train. You need a ticket to get on the train, and the ticket determines where you must get off. The ticket does not make the train, drive the train, power the train, determine what path the train will take, or create you as a passenger. Stuart Kaufmann (2000) described self-organising patterns and processes that seem to govern much of evolution. This self-organising criticality makes evolutionary and ecological systems subject to final causality in the same way as the mind/brain system is.

It seems that, in the course of evolution, features arise that are later co-opted for other purposes, or from features that seem to serve no purpose at all. These features are called preadaptations. This term was later abandoned for the alternative, exaptation, to avoid the implication of final causality, although the term preadaptation is still sometimes used. The ancestors of birds, for example, are said to have developed feathers for thermal regulation, which were later 
critical to flight. The wings of birds are said to have developed gradually, again as a means of thermal regulation.

A well-known example of an exaptation is the rapid evolutionary growth of the human brain, leaving spaces or spandrels, which were thought to have been later co-opted for language, art, commerce, war, and other functions (Buss et al., 1998). It should be noted that the large cranium of humans had a negative survival value. The pelvic orifice in the female had to be widened substantially to give birth, leading to death of the infant and/or mother in those that did not have a wide pelvic orifice. Notwithstanding, infant and maternal mortality in childbirth, as a result of delivery of a large cranium, would have remained high. In order for the enlarged cranium to be delivered through the pelvis, infants would have to be delivered at an early stage of development, making them unable to survive for a substantial period of time without protection and care, further increasing infant mortality. These changes would have to have co-evolved with the increasing size of the human brain. In terms of natural selection, there would have had to have been a clear survival and reproductive benefit of the enlarged brain, and brain size would have had to have been an adaptation offsetting these maladaptive features. This adaptive value would also have been sufficient to warrant co-evolution of other features. However, the rapid growth of the human brain was recognized as a preadaptation by Steven J. Gould (Buss et al., 1998), arguably the greatest evolutionary scientist of the twentieth century.

The eye is another complex organ that had to have evolved in stages. Stuart Kauffman (2009) noted that (p. 5) "the eye, or even a red light sensitive cell in the progeny of an organism with no light sensitivity, is a "blind teleology.'" Kauffman also states (p. 2) that "no finite set of laws may suffice to describe by entailment the evolution of the physical universe." Kauffman describes the preadaptation as fundamental to evolution, and goes on to say that (p. 3) "the evolution of the biosphere by Darwinian exaption, or 'preadaptations,' is not describable by sufficient natural law." He argues that the opportunity for adaptation is a "blind final cause," which is the missing "why" of Darwinian evolutionary theory. He argues in favor of a partially lawless and creative universe, such the 
evolution of the biosphere and of species is inherently unpredictable. If is my own opinion that the evolution of humans, as I understand it, as well as the very special conditions that apply to our planet, could not possibly be "inherently unpredictable," because if they were, they could not possibly have occurred. This being the case, I make no apologies for the belief that God created us in His (or Her) own image, through the process of evolution, as final cause.

If we accept the notion that evolution occurs through preadaptations, which are unpredictable in both origin and outcomes, but are somehow propagated in the biosphere by blind teleology or blind final cause, not describable by natural law, we must inquire as to how the blind teleology is referred to some future actuality. If the phenomenon is not referable to some future actuality, or possibility of actuality, how can it possibly be called a final cause? If there is connection between the phenomenon and some future actuality, in what sense is it blind? God produces final causes from the realm of the possible future, and such final causality would help explain the difficulties that we have in explanation of preadaptation and other evolutionary problems.

In the Universe as we know it, the highest expression of the Mind of God is in humanity. However, the Mind of God, the One Mind, is so far beyond the mind of any single person as to be inscrutable, and the upward evolution of the mental and spiritual nature of man will continue to limits we can only imagine. We are the final cause of the progressive leaps in evolution which have led our forbearers through successive levels of experience. The quantum observation that creates our reality is of the nature of experience, such that higher levels of experience over time are the motive force of evolution, moving forward in time. The movement of the subjective aim toward satisfaction of such high grades of experience is the formal cause of evolution, while natural selection is a mechanism serving that cause. 


\section{Conclusion}

The future is potential, known as such only to God. Humankind is faced with challenges as well as opportunities. We manifest in groups as we manifest as individuals. Some 200 years ago, the scientist Pierre Simon Laplace famously said regarding God that there was no need for such a hypothesis. Scientists today have much the same attitude. God must be causal to be relevant to science. God is necessary at the scientific level of description as the agent of final causality. Both God and final causality are strictly forbidden by orthodox science. We have argued here that there now is a need for God in our hypotheses, and that, defining God's functions on a Whiteheadean basis, the hypothesis of God has substantial explanatory power.

Quantum mechanisms for mind and consciousness are deficient in that the brain, although supporting a wealth of quantum processes on the level of the particle, and to a less degree on the atomic and molecular levels, cannot support the necessary level of quantum coherence, or even a relevant, sustained wave function. Quantum models must then resort to mind/brain interaction and mind/brain dualism, utilizing the hypothetical quantum theory of the observer. How can the observer be in the brain if the observer is the brain? The link between observation and actuality in quantum theory gives us a single Universe, honed out of the enormous realm of possibility, through the agency of a single God or One Mind.

Many of the objections from science and scientists to the integration of theology and science are based on deism, a notion inherited from classical physics. In the bridging of science and theology, we will need to develop a theology that suits the times, and this is, in large part, what we have attempted to do here. This has involved the synthesis of many disciplines, and I beg indulgence for being better versed in some of these disciplines than in others.

We now have need for the "hypothesis" of God, as a unifying principle, as the source of order, and as the final causality that makes science work. For God, process starts at the uppermost place on the mental pole, as a consciousness that is higher than our own, everywhere present. Perhaps part of the reason why Whitehead introduced God into his metaphysics was because, 
fundamentally, he needed God to complete it. Thus, the main section on God is near the end of Process and Reality. In the same sense, God is active, and not just a passive observer and so science will need God for its completion.

\section{Acknowledgements}

I am a retired psychiatrist, now living in New Jersey, where I was born. Many years ago, after reading Process and Reality, I became involved with the Center for Process Studies in Claremont. I am thankful to everyone in the Center who helped me to understand, to the extent that I had been able, the many aspects of process and the meaning of Process and Reality. I am especially thankful to John Cobb, who had been quite gracious. I recall telling Professor Cobb many years ago, that I read PR as a blueprint of the mind. He seemed to approve. This paper is an attempt to unravel that blueprint, and, perhaps, to make some contribution to a process metapsychology.

\section{References}

Böhm, D., \& Hiley, B. J. (1993). The undivided universe: An ontological interpretation of quantum theory. London and New York: Routledge.

Buss, D. M., Haselton, M. G., Shackelford, T. K., Bleske, A. L., \& Wakefield, J. C. (1998). Adaptations, exaptations, and spandrels. American psychologist, 53(5), 533-548.

Cobb Jr, J. B. (2008). Whitehead word book. sq.(trad. française légèrement modifiée: Lexique whiteheadien, Editions Chromatika, 2010, pp. 87-88). Cette caractéristique s'appliquant à tous les animaux dotés d'un système nerveux central.

Germine, Mark. (2011). The Book of Eli. Xlibris: Amazon Digital Services LLC.

Kauffman, S. A. (2000). Investigations. Oxford: Oxford University Press.

Kauffman, S. A. (2009). Towards a Post Reductionist Science: The Open Universe, Physics Archives. Retrieved from arXiv:0907.2492 [physics.hist-ph]. 
Neumann, E. (1954). The Origins and History of Consciousness. Princeton: Princeton University Press.

Stapp, H. P. (2007). Mindful Universe: Quantum Mechanics and the Participating Observer. Berlin: Springer-Verlag.

Trischler, A. P., \& D'Eleuterio, G. M. (2016). Synthesis of recurrent neural networks for dynamical system simulation. Neural Networks, 80, 67-78.

Whitehead, A. N. (1927). Religion in the Making: Lowell Lectures 1926. New York: Macmillan.

Whitehead, A. N. (1968). Modes of Thought. New York: The Free Press.

Whitehead, A. N. (1978). Process and Reality. In D. R. Griffin and D. W. Sherburne (Ed.), Corrected Edition. New York: Free Press, 47, 277. 